\title{
Magic in pieces: an analysis of magic trick construction using artificial intelligence
}

\section{as a design aid}

Abbreviated title: Magic in pieces

Howard Williams, Peter W. McOwan

School of Electronic Engineering and Computer Science,

Queen Mary University London,

Mile End Road,

London E1 4NS

\begin{abstract}
Artificial intelligence (AI) techniques are successfully used for various design and optimisation tasks, and have previously been applied to design magic tricks. A magical effect is an event that the observer perceives as being outside the normal physical rules of the world. In this paper we revisit an AI framework that has been used to produce a two dimensional interlocking piece jigsaw design with two simultaneous solutions, one showing twelve identical shapes, the other only ten, giving rise to the believable illusion of the shapes vanishing. To achieve this effect the design framework enables the combination of both geometric optimisation constraints and constraints derived from empirically determined psychophysical and cognitive investigations.

We further discuss the human factors that affect an observer's perception of a magic trick, and how these observations can be used in a computational optimisation process. We also provide a more detailed analysis of the algorithms used to generate the magical effects.
\end{abstract}




\section{Introduction}

The use of computers as creative entities has been explored in various contexts (Boden 1998, Bentley 2002). Using Artificial Intelligence (AI) techniques to synthesise and optimise existing ideas and forms has resulted in some notable successes in the field of computer gaming where AI techniques have been used to optimise both elements of the games themselves (Liaw 2013) and the overall entertainment value provided by the games (Yannakakis 2007). Product design has also benefited from the introduction of computational optimisations into the process (Roy 1995). Marvin Minsky has outlined the potential future merging of science, art and psychology (Minsky 1993); our work is a concrete instantiation of these ideas. In this current paper we expand on work previously described in (Williams 2014). Here we extend the discussions to further detail the human factors that affect an observer's perception of a magic trick, and how these observations can be used in developing a unified computational optimisation process. We also provide a more detailed and critical analysis of the algorithms used to generate our magical effects. Specifically, an optimisation system based on a Genetic Algorithm (GA) (Goldberg 1989, Deb 2002) and a Rectangle Packer (Lodi 2002), that is configured with psychophysical observations (Gescheider 1997).

Much work has been done in recent years to study human perceptual systems by observing the ways in which magicians exploit and manipulate them during the performance of magic tricks (Kuhn 2008). By examining these cognitive processes, and applying computational methods to deal with the large number of variables involved, we have been able to create novel magical effects: events that the observer perceives as being outside the normal physical rules of the world (Lamont 1999). A vanish is one of a number of basic effects that magicians are able to achieve (Lamont 1999), and the one that we exploit here.

The jigsaw is based on a category of effects that rely on The Principle of Concealed Distribution (Gardner 1956): the geometrical redistribution of one shape among a number of 
others such that the magnitude of increase in the area of the remaining shapes is imperceptibly small. The more shapes that are used the smaller the increase. An early use of this principle is the DeLand paradox (Gardner 1956), as shown in Figure 1. In this trick an image showing a number of rectangles is sliced horizontally and vertically, dividing the image into three pieces; the top or bottom half all one piece, the other half divided in two in some ratio. The two pieces that make up the top or the bottom half are then swapped over (translated in the x-dimension), recombining the rectangles in a way that leaves one fewer than before, all of the same perceived length but physically smaller than the rectangles in the original image. In our work two rectangles vanish, and the pieces are translated and rotated in two dimensions.

Observing that the DeLand paradox can be made up as a two dimensional jigsaw allows for greater flexibility in how the shapes can be positioned and redistributed, while simultaneously increasing the sense that something physically impossible has happened. In terms of a combinational problem, there are parallels to electronic circuit design.

The jigsaw produced by the AI system, has, unusually, two solutions: one showing an image depicting twelve identical vertically orientated rectangles, the other showing ten, crucially, imperceptibly longer, horizontally arranged rectangles. Moving from one solution to the other elicits a magical vanish, or, in other words, on casual observation, a seemingly physically impossible event. An example jigsaw is shown in Figure 2, where twelve bolts of lightning (in place of rectangles) contained between twelve pairs of hands in the image on the left become ten bolts of lightning contained between twelve pairs of hands in the image on the right (using the same jigsaws pieces, arranged differently). This basic schema can be depicted in various contexts for the important purposes of storytelling during magic tricks (Ortiz 1994).

The experience of viewing a magic trick is complex, and varies from person to person. Defining what it is that makes a magic trick seem magical, is difficult. The approach taken here 
is to deconstruct the perceptual and cognitive elements that an observer experiences during a particular trick (the jigsaw), and to assume that their combination in some way allows the trick to be effective. According to Lamont (Lamont 2013), a vital technique that allows a magic trick to be viewed as such is for the performer to construct the events in a way, to frame them, such that when the supposedly magical event occurs (here, vanishing objects) a so called pseudoexplanation is available to the observer that explains the events. This explanation does not actually explain how the events have occurred (the method behind the trick), thus nullifying any magical effect, but instead serves as a mental handle which the observer may hold on to as a way of ceasing any further inquiry into the real method at work. Often, the pseudo-explanation will revolve around some implied ability that the performer may have set themselves up as possessing; in the case of the jigsaw puzzle, the pseudo-explanation is in fact provided by the story that is narrated during the trick's exposition, and in fact any magical powers are bestowed upon fictional characters in this story. The effect of this narrative on the observer is analysed empirically here, via experiments in which the trick is performed both with and without the narrative, thus in a way that both provides and withholds a kind of pseudo-explanation for the observer to hold on to.

Viewing a magic trick activates brain areas that are involved in general cognitive conflict (Parris 2009). The jigsaw trick presented here undermines the concept of object permanence in an observer's mind - an extant object should not be able to simply disappear. The method of the trick relies on the imperceptible change in length of the rectangular objects on view; thus, in its final configuration, there is no readily apparent explanation for the object's disappearance, presumably activating the brain's conflict centres. This, in combination with the previously mentioned narrative, creates a compelling magical story for the observer to, in a sense, go along with. As we shall see, merely performing the steps of this type of trick mechanically results in a reduced effect for the observer. 
Bearing in mind that this jigsaw is intended to be used as a magic trick, i.e. performed as an effect for an observer, it should be noted that there are conflicting optimisation constraints on both the perception and performance of the trick. For the observer, the clearest effect occurs when the jigsaw is comprised of many pieces, but there are only a few rectangles on its surface to count. More pieces makes it less clear that the pieces are being reconstituted in a different configuration, while fewer rectangles makes it less likely that the observer will be able to explain the vanishing by assuming that they have merely lost count of the number of rectangles. Conversely, for the performer, error free construction and undetectable length increase of the rectangles is enabled with fewer pieces, but more rectangles. Fewer pieces makes it less likely that the performer will fumble the reconstruction, and have to re-arrange pieces, thus breaking the narrative flow. The more rectangles there are, the smaller the increase in size of the remaining rectangles, making the size increase less likely to be detected.

We have performed three experiments in an attempt to encapsulate the performance and perceptual factors involved in the trick. These factors make up the psychophysical components of the fitness function used by the GA.

One, an experiment to determine how many rectangles can be effortlessly counted by an observer. Second, a trial to determine how many jigsaw pieces are likely to cause construction problems for a performer of the trick.

Third, we investigated the magnitude of length increase of the rectangles that could pass undetected. The rectangles on the jigsaw can be positioned and oriented in different configurations while still satisfying the geometric constraints that produce the apparent vanish. For example, the first jigsaw might show an image containing eight vertical rectangles and four horizontal rectangles, while the second image shows a similarly mixed set of vertical and 
horizontal rectangles. Equally, the rectangles could be all vertical in the first image, and all horizontal in the second - we expected this type of configuration to allow the vertical-horizontal illusion to take effect (Robinson 1998): the overestimation by the human brain of vertical line lengths simultaneously compared with identically sized, but orthogonally oriented, stimuli. We have empirically determined the magnitude of length increase that will pass undetected, on casual observation, under various orientation conditions for stimuli presented at different times (to mimic the mechanics of the jigsaw trick).

We have incorporated the data from these three experiments into the fitness functions used by the GA to design a psychophysically compelling illusion that is optimal for both performance by a magician, and perception by an observer.

Finally, we performed an experiment to measure the efficacy of our optimisations on the overall experience of the trick. For this, a version of the trick with lightning bolts standing in as the rectangular objects was used; the use of these graphics tied in to the story told during the trick's performance. This experiment also examined the impact of narrative on performance and meaning to determine whether a magic trick reduced purely to mechanical actions can be as effective as one with a story to follow.

There is an intractable combinatorial explosion of possibilities for jigsaw designs, only some of which satisfy our constraints. Genetic algorithms are excellent optimisers (Goldberg 1989). Their use as a design tool, as a way of replacing a human designer for constrained problem domains, has been investigated by a number of researchers (Bentley 2002). As outlined, our GA combines geometric constraints with psychophysical constraints derived from our experimental work on rectangle length perception, jigsaw construction, and cognitive load induced by counting shapes. The geometric problem inherent in jigsaw design has similarities with combinational electronic circuit design, a problem domain identified and investigated first 
by Louis with his work on structure design (Louis 1991), and followed up and expanded by a number of others, including: Arslan's efforts on the structural synthesis of VLSI circuits (Arslan 1996) and Coello's GA automated process to minimise the number of gates used by a circuit (Coello 1999

We implemented an automated system that is capable of synthesising the various geometric and perceptual elements we have discussed to design novel jigsaw tricks to flexible specifications. The system can be configured with the appropriate psychological constraints to guide its design process: maximising the ease of performance and concealment of underlying mechanics, while minimising the cognitive effort required by the observer to experience the magical effect. 


\section{The Algorithm}

As part of the algorithm used, a multi-objective bit-encoded NSGA-II (Deb 2002) derived genetic algorithm (GA) was formulated to design the jigsaw, performing tournament selection (Goldberg 1989).

Multi-objective optimisation techniques are applied to problems, such as the jigsaw produced here, where conflicting constraints mean there is not necessarily a single solution where each objective is optimal; a balance must be struck. Where fitness functions contain conflicting constraints there can be any number of groups of Pareto optimal (non-dominated) candidate solutions, termed Pareto fronts, at any point in the optimisation process - a non-dominated solution is a solution where none of the component fitness values can be improved without diminishing some of the other values.

The NSGA-II algorithm introduces the crowded-comparison operator, used as a metric to compare candidate solutions to each other based on the rank of each solution and the density of other nearby solutions.

Rectangle packing (Lodi 2002) techniques, guided by the GA, were also used in the algorithm to aid placement and rearrangement of the jigsaw pieces.

\section{Algorithm - NSGA-II derived genetic algorithm using rectangle packing techniques}

Objectives used in fitness evaluation:

1. Area of first and second jigsaw solution covered by generated pieces (Geometric constraint)

2. Number of pieces that are fully connected by jigsaw lugs in first and second jigsaw solution (Geometric constraint)

3. Number of rectangle fragments that connect to form complete rectangles of correct size in each jigsaw (Geometric constraint)

4. Number of rectangles in each jigsaw (Geometric constraint) 
5. Spatial distance of rectangles from configurable points on the jigsaws (Geometric constraint)

6. Total number of pieces, scored from a scale mapped from experimental data (Psychophysical constraint)

7. Total number of rectangles, scored from a scale mapped from experimental data (Psychophysical constraint)

8. Rectangle orientation score for each jigsaw, scored from a scale mapped from experimental data (Psychophysical constraint)

Candidate jigsaw solutions are typically encoded as approximately 200-bit entities, with differing amounts of bits used for more or less complex puzzles.

- BEGIN

- INITIALISATION

- A number of candidate jigsaw solutions are randomly generated into population $\mathrm{P}$ (size 250)

- Candidates P are sorted and ranked into non-dominated fronts (NSGA-II) based on fitness function (geometric and psychophysical constraints)

- Tournament selection (based on fitness rank), mutation (per bit mutation rate $=$ 0.004 ) and crossover (rate $=0.9$ ) operators are applied to $P$, creating a new population of candidates $Q$ (size 250)

- LOOP FOR X GENERATIONS

- A combined population R (size 500 ) is formed from $P$ and $Q$ and is subsequently sorted and ranked into non-dominated fronts

- A new population $\mathrm{P}$ of size 250 is created by taking the fittest candidates from $\mathrm{R}$

- Tournament selection (based on NSGA-II crowded-comparison operator), mutation and crossover operators are applied to $\mathrm{P}$, creating a new population of candidates $\mathrm{Q}$ of size $\mathrm{R} / 2$

- ENDLOOP

- END

The algorithm converges to solutions in less than fifty generations, more often in less than fifteen - the number of pieces and number of rectangles increases the complexity. The 
computation time to design the example featured was approximately two minutes on a desktop PC with an Intel Core i5 processor.

\section{Experimental Methods}

The absolute threshold at which the growth in length of the rectangles would be perceptible, as used by the algorithm in its fitness function, was determined experimentally. The participants $(n=156)$ were shown pairs of sequentially presented images, separated by a blank screen. Each pair consisted of an image of six rectangles of a certain width and length, of vertical, horizontal or mixed orientation, shown for one and a half seconds, followed by a blank screen for one second, followed by a second image of six rectangles of vertical, horizontal or mixed orientation. All rectangles were randomly positioned on screen with none overlapping. The change in orientation of the rectangles was designed to determine if the vertical-horizontal illusion would be effective using sequential stimulus interrupted by a blank screen, and to investigate the general effect of orientation on length perception. The group of rectangles in the second image would either be the same length as in the first image, or would increase by a certain percentage. The increase ranged from $0 \%$ to $30 \%$, in $5 \%$ increments. A pair depicting a certain percentage length increase was shown to the participant ten times, with a random order of presentation - the Method of Constant Stimuli (Laming 1999). The participants were asked only to determine if the lengths of the second set of rectangles had increased in comparison with the rectangles in the first image; a yes or no. The precise threshold was calculated by regression fitting a line to the data points, allowing the amount of size increase that could be detected $50 \%$ of the time (the absolute threshold) to be calculated.

An experiment was run to determine the time taken for subjects $(\mathrm{N}=30)$ to count the number of rectangles on a screen; during each presentation the subject would see between 2 and 16 rectangles. The rectangles were presented in a vertical, horizontal or mixed orientation. The 
rectangles remained on screen until the subject indicated how many there were, at which point the next set were shown. The number and orientation of rectangles in each set was randomly generated over the course of the experiment, with each subject viewing five of each combination.

A pilot study was conducted during which subjects $(\mathrm{N}=5)$ were asked to assemble, as quickly as possible, blank jigsaw pieces into a square shape. They were given differently numbered sets of pieces in a random order, and the time taken to complete the jigsaw was recorded.

To determine the overall rating of the jigsaw trick, subjects $(\mathrm{N}=100)$ were shown a video of a performance and asked to rate their overall enjoyment of it on a scale with the following values:

”Hated”, ”Disliked”, ”Neutral”, ”Liked”, ”Loved”, with corresponding values from zero to four. Each participant viewed only one performance. The performance was randomly selected from nine different performances: four versions of the jigsaw trick, one showing vanishing rectangles with a narrative, another with vanishes but no narrative, and two versions with no vanishes: one with narrative, one without. Five additional performances could be viewed, each depicting a skilled magician performing a classic magic routine of around the same length as the jigsaw trick (ranging sixty to ninety seconds). The five tricks were: a vanishing cup routine, a vanishing cloth in hand, a floating piece of paper, a small to big coin transfer, and a broken cigarette fixed in hand trick.

\section{Experimental Results}

We experimentally determined the threshold at which the growth in length of rectangles is perceptible, investigating the effect of orientation. We investigated the absolute threshold of change in size perception for people shown two successive images, containing rectangles orientated vertically, horizontally or a mixture of both in each image. In line with previous work 
showing the vertical-horizontal illusion, the greatest absolute threshold value of $21.1 \%$ was found when subjects were shown an image containing all vertical rectangles, followed by an image containing all horizontal rectangles.

To evaluate cognitive load produced by the observer of the trick being required to count the number of rectangles on the puzzle, we determined experimentally that the rate at which subjects were able to count rectangles on a screen rose linearly with the number shown. This provides a simple scale for the optimiser to use. A smaller cognitive load for the observer for this type of task is desirable at it reduces the possibility that they will explain the vanishing of the objects with the idea that they have lost count. Further, if they can easily count the number of rectangles they will be more easily able to listen to and follow the narrative being conveyed by the performer.

Similarly, for the performer, a trick with too many pieces could take too long to assemble, and be prone to error, disrupting the performance. We show that the time taken for subjects to assemble blank jigsaw pieces into a square shape probably becomes highly variable beyond eight pieces. When more pieces were introduced some subjects found themselves in great difficulty.

We experimentally evaluated the quality of the magical effect in comparison with classic magic tricks on a scale, mapped numerically, of: Hated (0), Disliked (1), Neutral (2), Liked (3), Loved (4). The overall rating of the jigsaw with a full narrative describing the trick was, on this scale, 2.41. A version of the trick with no narrative scored 2.17. A version of the jigsaw that had no vanishing elements (hence, no magical effect at all) and no narrative rated 1.53, while a version with no vanishes but a full narrative rated 1.83 . The classic magic tricks performed by a skilled magician were rated between 2.26 and 2.86 . 
A one-way between subjects ANOVA was conducted to compare the effect of trick seen on enjoyment rating for tricks seen in [Jigsaw NO trick NO narrative], [Jigsaw NO trick WITH narrative], [Jigsaw WITH trick WITH narrative], and [Jigsaw WITH trick NO narrative] conditions. There was a non-significant effect of trick seen on enjoyment rating at the $\mathrm{p}<0.05$ level for the four conditions $[\mathrm{F}(3,96)=2.515, \mathrm{p}=0.06]$. Initial results suggest using more subjects in the experiments may result in a significant difference between tricks seen. 


\section{Discussion}

We have shown that certain geometrical properties allied with certain attributes of the human visual system allow for the discovery, via the application of an artificial intelligence algorithm, of a new conjuring trick in the form of a jigsaw. More specifically, it has been shown that the underlying method, the Principle of Concealed Distribution, can be made imperceptible as the increase in length of the rectangles on display during the horizontal presentation of the jigsaw is less than the $21.1 \%$ threshold at which a viewer would be able to perceive any change. We believe our techniques show how AI methods allied to human perceptual observations can be used as designers of novel conjuring tricks, and that these tricks provide insight into certain high level human perceptual and cognitive processes. The designed jigsaw puzzle has been made into a real product that has sold out each of its production runs at a popular magic shop in London. This provides an additional real world method for validating the work: the output of the design system has been successfully sold to practicing magicians who, in the act of purchase and performance of the trick, attest to its entertaining magical properties.

\section{Reference}

- Arslan, T., Horrocks, D. H. \& Ozdemir, E. Structural cell-based VLSI circuit design using a genetic algorithm. In ISCAS'96: 1996 IEEE International Symposium on Circuits and Systems: Circuits and Systems Connecting the World, Volume 4, 9-6 (IEEE, 1996).

- Bentley, P. Creative Evolutionary Systems (Morgan Kaufmann Publishers Inc., 2002).

- Boden, M. Creativity and artificial intelligence. Artificial Intelligence, Volume 103, Issues 1-2, Pages 347-356 (1998).

- Coello, C. A. C., Christiansen, A. D. \& Aguirre, A. H. Use of evolutionary techniques to automate the design of combinational circuits (1999). 
- Deb, K., Pratap, A., Agarwal, S. \& Meyarivan, T. A fast and elitist multi-objective genetic algorithm: NSGA-ii. Evolutionary Computation, IEEE Transactions on 6, 182197 (2002).

- Gardner, M. Mathematics Magic and Mystery (Dover Publications, 1956).

- Gescheider, G. Psychophysics: the fundamentals (Lawrence Erlbaum Associates, 1997).

- Goldberg, D. Genetic Algorithms in Search, Optimization, and Machine Learning (Addison- Wesley, 1989).

- Kuhn, G., Amlani, A. A. \& Rensink, R. A. Towards a science of magic. Trends in Cognitive Sciences 12, 349 - 354 (2008).

- Laming, D. \& Laming, J. Hegelmaier: On memory for the length of a line. Psychological Research 54, 233-239 (1992).

- Lamont, P. Extraordinary beliefs: A historical approach to a Psychological problem. Cambridge: Univesity Press (2013).

- Lamont, P. \& Wiseman, R. Magic in Theory (University of Hertfordshire Press, 1999).

- Liaw, C. \& Wang, W. \& Tsai, C. \& Ko, C. \& Hao, G. Evolving a team in a first-person shooter game by using a genetic algorithm. Applied Artificial Intelligence 27, 199-212 (2013).

- Lodi, A., Martello, S. \& Monaci, M. Two-dimensional packing problems: A survey. European Journal of Operational Research 141, 241 - 252 (2002).

- Louis, S. \& Rawlins, G. J. E. Designer genetic algorithms: Genetic algorithms in structure design. In Proceedings of the Fourth International Conference on Genetic Algorithms, 53-60 (Morgan Kauffman, 1991).

- Minsky, M. The future merging of science, art and psychology. Applied Artificial Intelligence 7, 87-108 (1993).

- Ortiz, D. Strong Magic (Ortiz Publications, 1994). 
- Parris, B. A., Kuhn, G., Mizon, G. A., Benattayallah, A., \& Hodgson, T. L. Imaging the impossible: An fMRI study of impossible causal relationships in magic tricks. Neuroimage, 45(3), 1033---1039 (2009).

- Robinson, J. O. The Psychology of Visual Illusion (Dover Publications, 1998).

- Roy, U. \& Bharadwaj, B. \& Sarathy, S. \& Graham,P. Development of an intelligent product design system: integration strategies. Applied Artificial Intelligence 9, 563-585 (1995).

- Williams, H., McOwan, P.W., Magic in the machine: a computational magician's assistant. Frontiers in Psychology, 5, 01283 (2014)

- Yannakakis, G \& Hallam, J. Towards optimizing entertainment in computer games. Applied Artificial Intelligence 21, 933-971 (2007).

Ethics approval: QMREC2011/94 - Perception of computerised psychophysical stimuli

Acknowledgements: We thank the Engineering and Physical Sciences Research Council (EPSRC).

Competing Interests: The authors declare no competing financial interests.

Correspondence: Howard Williams. (email: h.williams@qmul.ac.uk). 

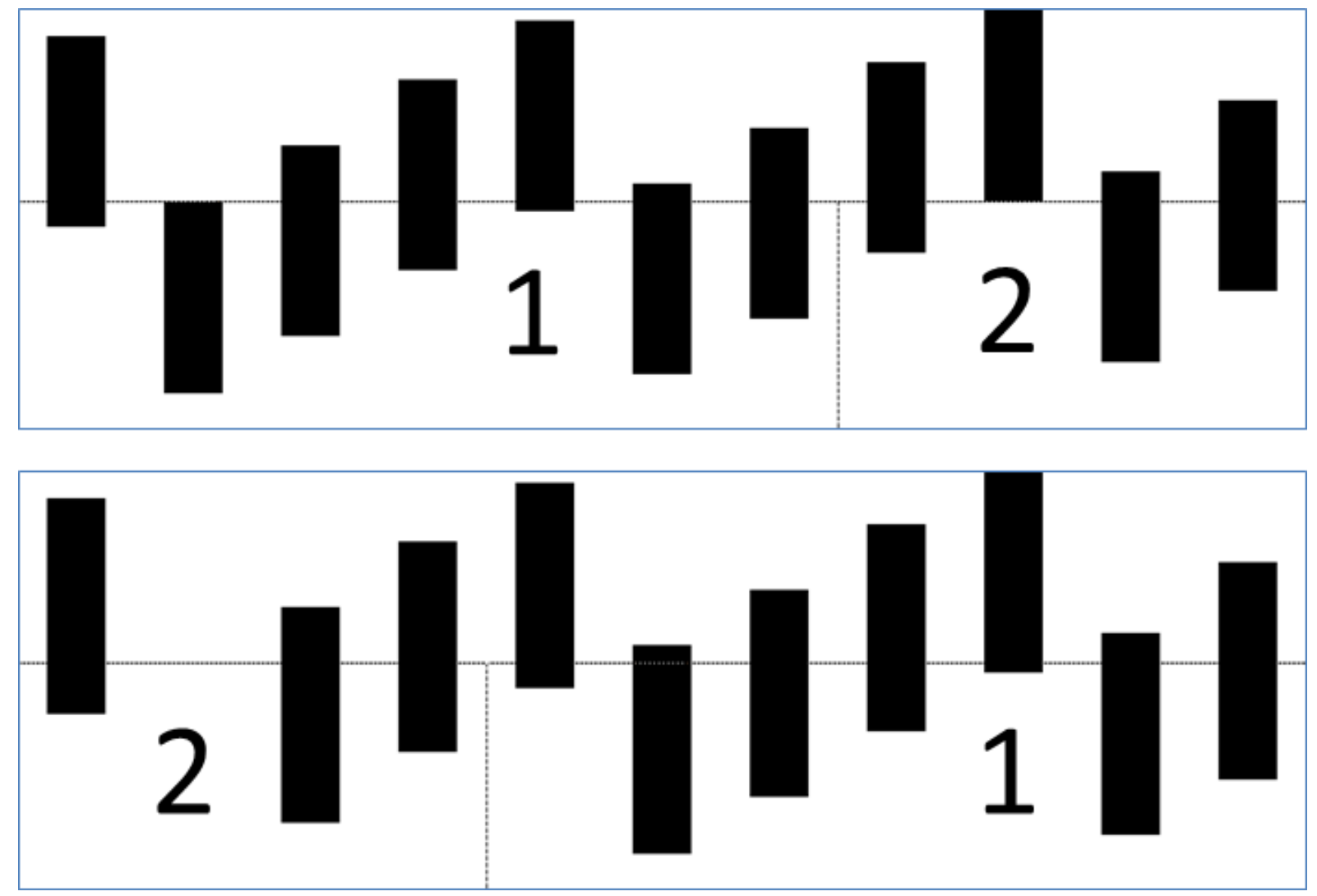

Figure 1. The DeLand paradox. The top image depicts eleven rectangles, the bottom, after swapping pieces 1 and 2, only ten. 


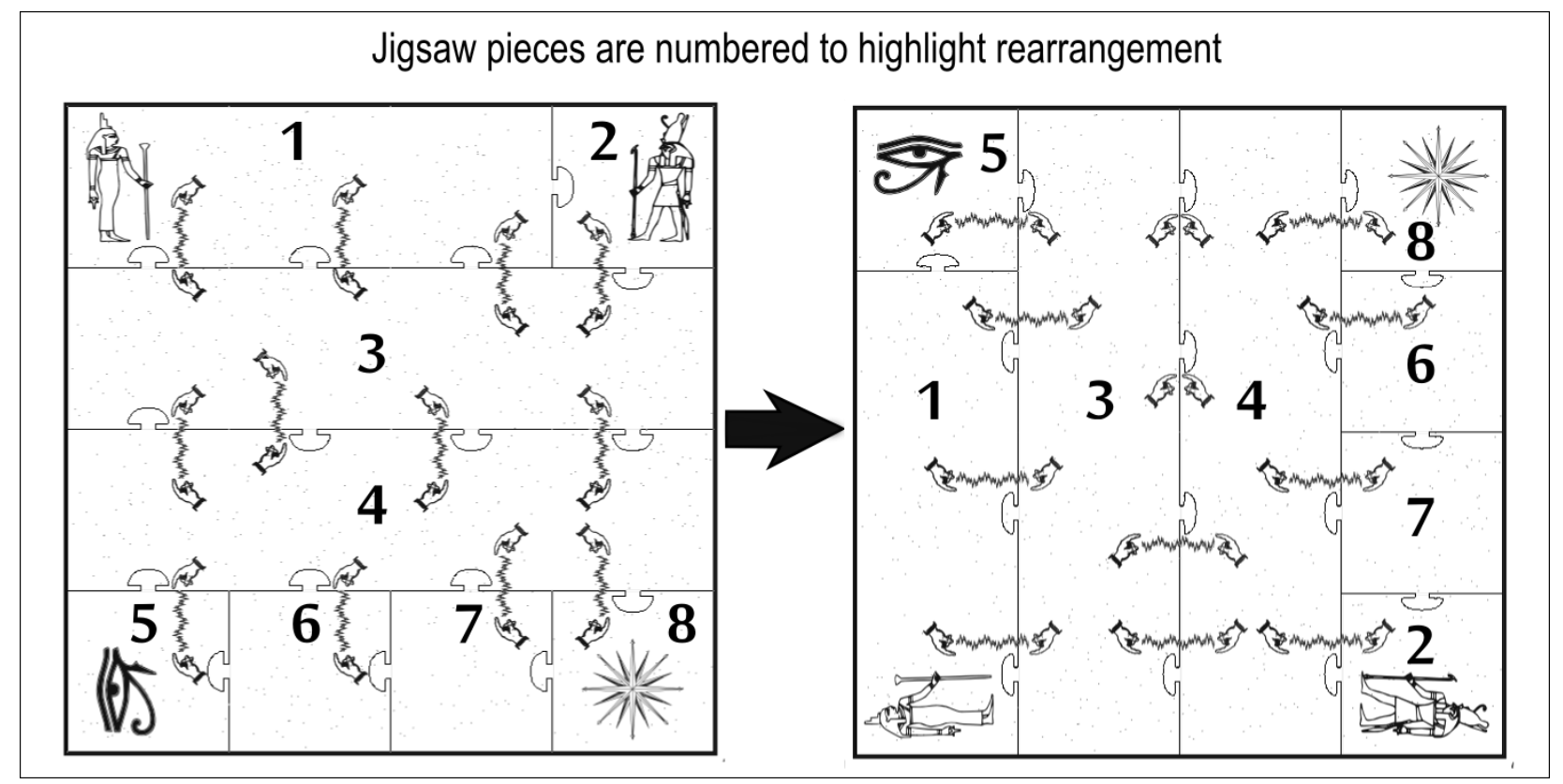

Figure 2. The designed jigsaw; an Egyptian themed trick is shown. The pieces are numbered to highlight rearrangement. The image on the left shows twelve lightning bolts, the image on the right only ten. Note the two vanished lightning bolts between the two pairs of hands shown on pieces 3 and 4 in the image on the right. The jigsaw pieces used are identical in each image. 


\section{Design process}
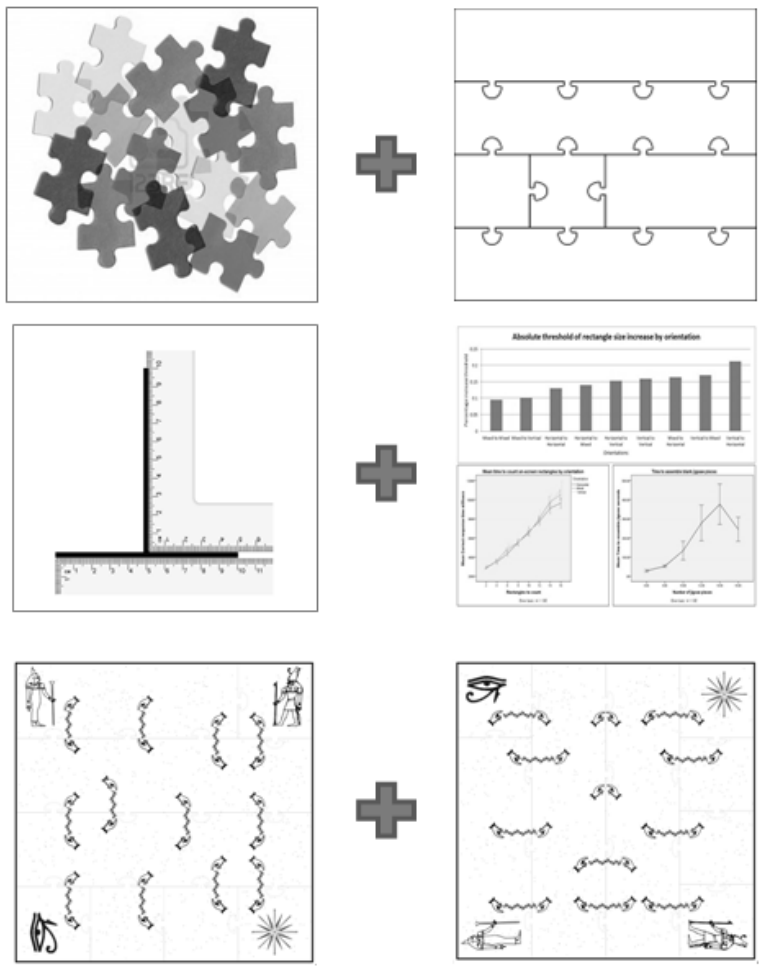

Al generates, encodes and seeds a population of candidate jigsaw solutions.

Each jigsaw's fitness is evaluated according to psychophysical criteria.

Evolutionary processes produce increasingly fit offspring over many generations.

The algorithm evolves a solution that satisfies a set of psychophysical constraints.

Figure 3. An artificial intelligence system, based on a Genetic Algorithm (GA), configured with psychophysical constraints evolves a perceptually optimal solution based on empirical data. 


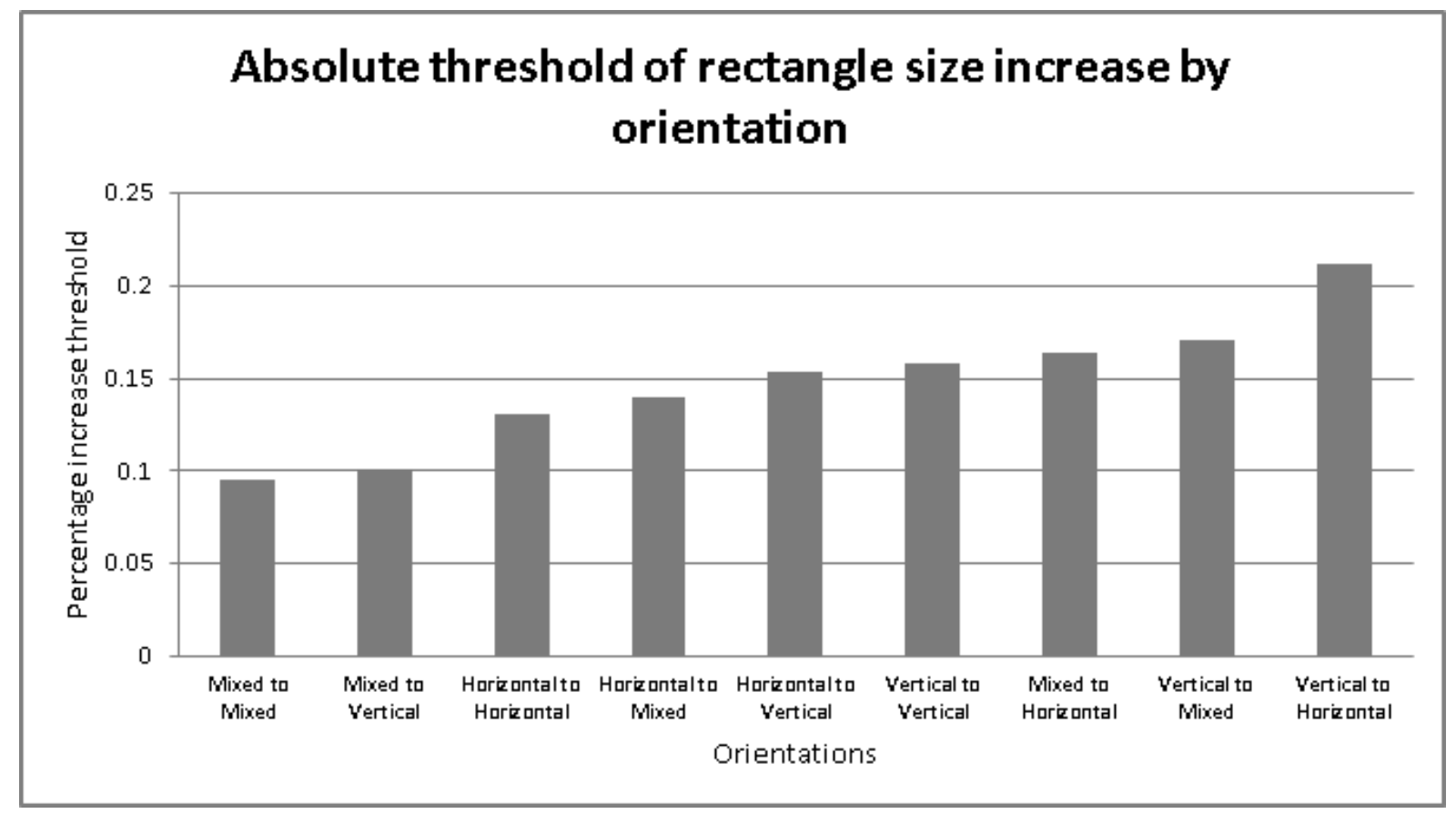

Figure 4. The absolute threshold of length increase of a rectangle, the point at which the change is noticeable, is highest when all rectangles are shown vertically, and then rearranged to be horizontal, confirming the vertical-horizontal illusion. 


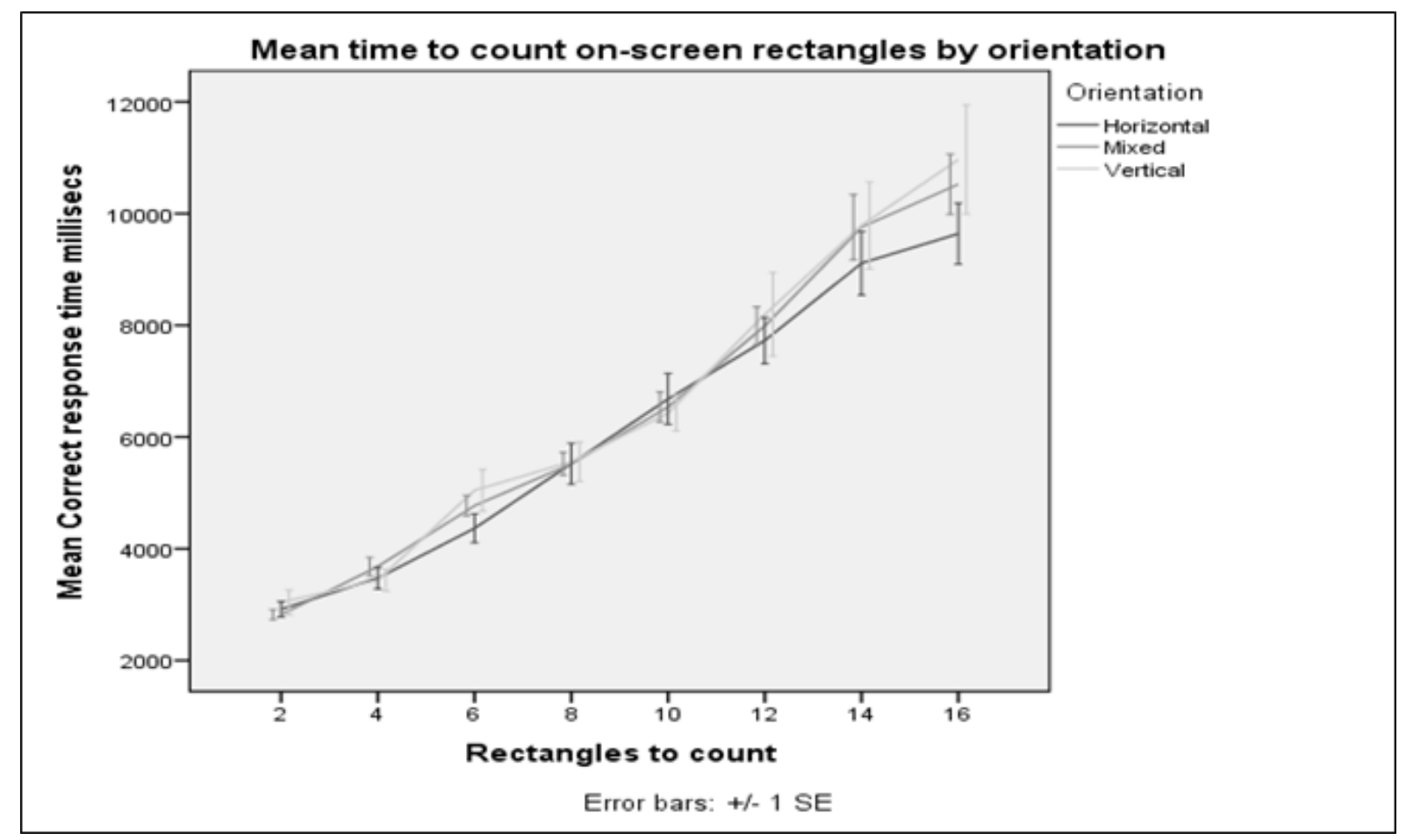

Figure 5. The time taken to count a set of rectangles rises linearly. Data points with standard error bars are shown. 


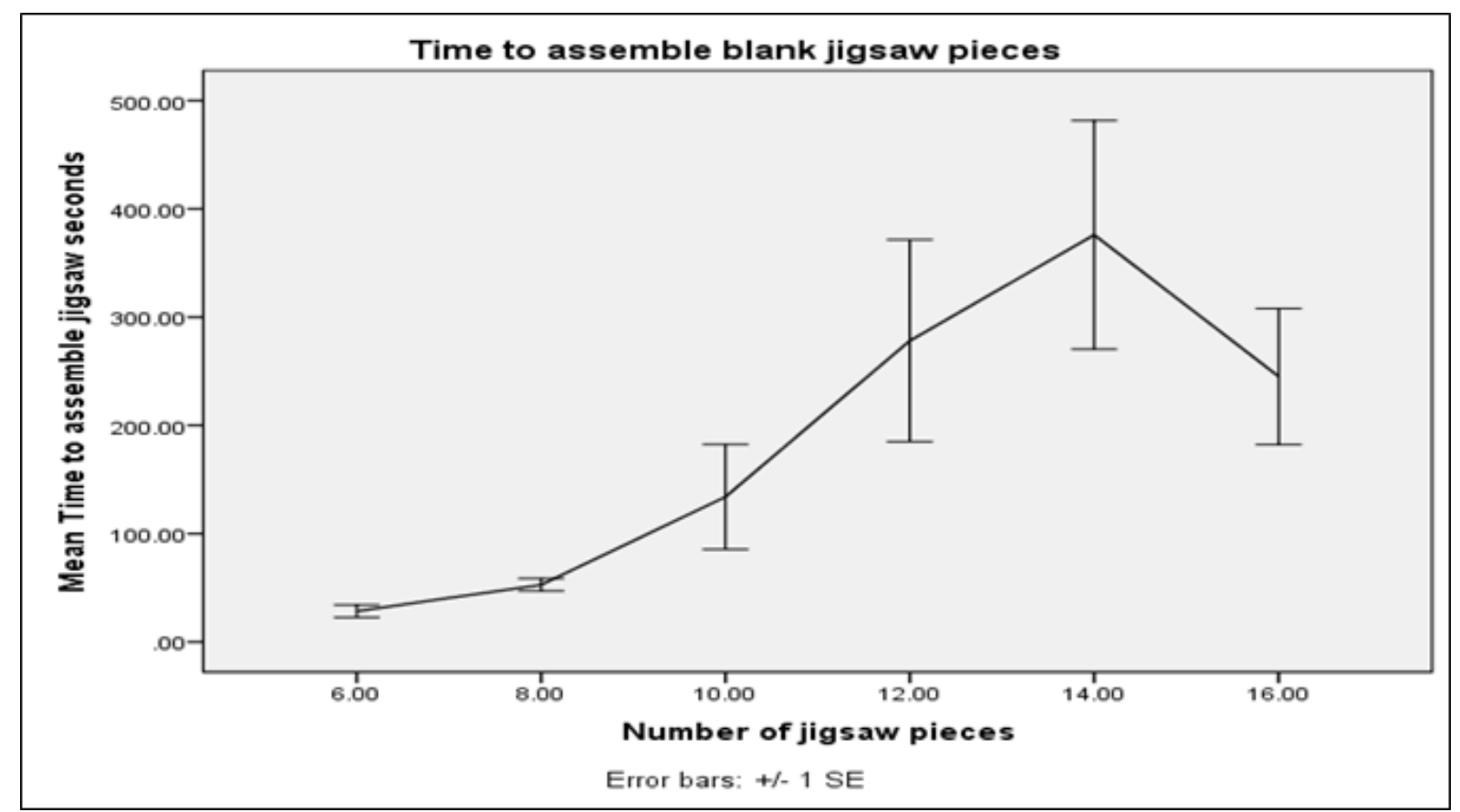

Figure 6. Asking subjects to assemble blank jigsaws with more than eight pieces showed high variability in time taken to complete the task; eight or fewer pieces should cause few construction issues. Data points with standard error bars are shown. 


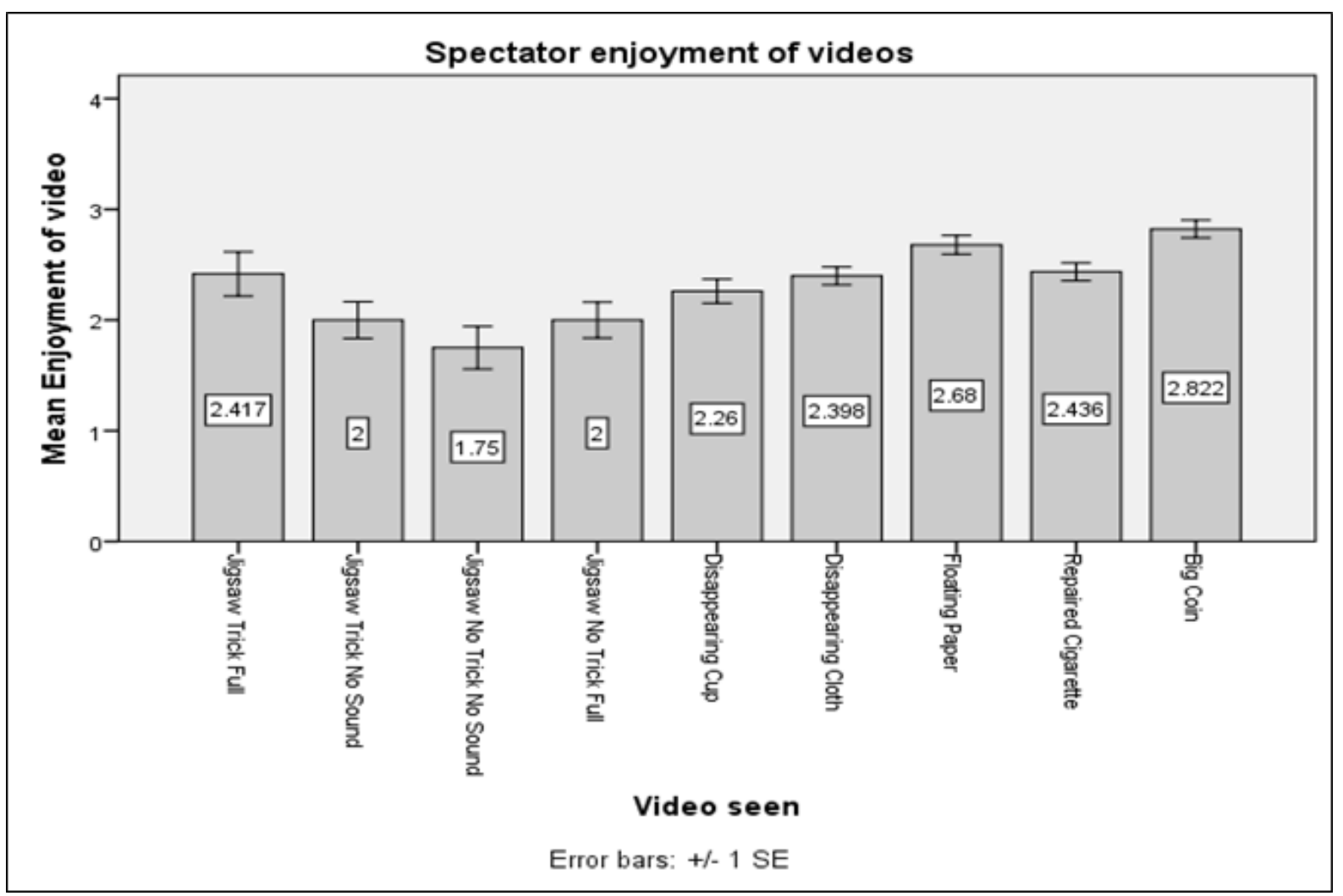

Figure 7. The designed trick was tested along with examples of classic magic tricks performed by a skilled magician. Users shown videos of the tricks were asked to rate their enjoyment on a five point scale from 'Hated it' to 'Loved it'. Variations of the jigsaw trick with and without a narrative were also shown to subjects, along with two variations where no rectangles vanished. The full trick with a narrative scored comparably with the classic magic tricks. Data points with standard error bars are shown. 